\title{
Orthosurgical management of odontome-associated maxillary central incisor impaction
}

\begin{abstract}
Odontomas are hamartomous lesions or malformations rather than true neoplasms and are frequent cause of impaction, delayed eruption of permanent teeth or retention of primary teeth. This case report describes treatment of compound odontoma in a twenty-year-old male presented to a postgraduate orthodontic clinic with chief complaint of space with respect to upper jaw on right side. Radiographically, a compound odontoma was present, which has resulted in failure of eruption of the permanent upper right central incisor. A sequential approach of surgical removal of odontome, surgical exposure and orthodontic traction of the impacted tooth with closed eruption technique resulted in proper incisor positioning. Close monitoring and interdisciplinary management during the treatment led to a successful esthetic result, with good periodontal health and functional occlusion
\end{abstract}

Volume 2 Issue I - 2015

\author{
Sushma Dhiman, Saba Khan, Sandhya \\ Maheshwari, Sanjeev K Verma, Mohd Tariq \\ Department of Orthodontics and Dentofacial Orthopedics, \\ Ziauddin Ahmad Dental College, India
}

Correspondence: Saba Khan, Department of Orthodontics, Ziauddin Ahmad Dental college, AMU, Aligarh 20200I, Uttar Pradesh, India, Tel 9997198983, Email apple.saba@gmail.com

Received: November 03, 2014 | Published: December 6, 2014

Keywords: odontogenic tumors, compound odontoma, delayed tooth eruption, orthodontic extrusion, forced eruption

\section{Introduction}

The term "Odontoma" was coined by Paul Braco in 1867, to define tumors formed as dental hamartomas of odontogenic origin. ${ }^{1}$ WHO defined this lesion as "a malformation in which all the dental tissues are represented, individual tissues being mainly formed but occurring in more or less disorderly pattern." Odontoma is probably the most common type of odontogenic tumor or hamartomas ${ }^{2}$ which histologically comprises of a complex picture of differentiated epithelial, mesenchymal cells which may be derived from ameloblasts or odontogenic epithelium..$^{3-5}$ Hence, an odontoma is a benign mixed tumor and differs from a true neoplasm. ${ }^{6}$

The etiology behind odontoma remains unclear, local trauma or infection has been implicated as a source of odontoblastic hyperactivity. Alterations in genetic components, which may be associated with syndromes like Hermann's syndrome and Gardner's syndrome, also leads to odontome formation. ${ }^{7}$ Usually odontomas are asymptomatic ${ }^{8-9}$ and are usually discovered during routine radiographs. Clinically, it presents with a delay in exfoliation of primary tooth or delayed eruption of the succedaneous teeth.

Developmentally, odontome can be of epithelial or connective tissue origin, it may also present as a composite mass comprising of both epithelial and mesodermal derivatives. ${ }^{10}$ They are clinically classified as complex or compound odontome. A compound odontome presents as a rudimentary tooth, with classified tissues arranged orderly into layers of enamel, dentin and pulp. ${ }^{11}$ Contrasting feature of complex odontome is its haphazard arrangement of dental tissues, with no morphologic similarity between the odontome and actual tooth structure. Commonly, compound variety of odontome is observed. ${ }^{12}$ Compound odontome have a propensity for anterior region of the jaw while complex odontome are usually seen in the posterior region. We present a case of maxillary central incisor impaction associated with a compound odontome, its radiographic features and ortho-surgical management.

\section{Case Presentation}

A 20year old young adult came to the Department of Orthodontics and Dento facial Orthopedics complaining of spacing with respect to the upper right front tooth. On general examination and medical history evaluation, it was found that the patient was apparently healthy. Patient revealed history of prolonged retention of 51 which was extracted by a dental practitioner five years back in an anticipation of eruption of 11. Patient gave a history of root canal treatment with respect to 12 , performed six months back as it the tooth was non vital.

\section{Clinical presentation}

The patient had apparently symmetrical, mesoprosopic face and balanced facial pattern. On intra-oral examination it was found that the patient had Class I canine and molar relation bilaterally, accompanied by an edentulous span in relation to right maxillary central incisor region (Figure 1). The overlying mucosa was apparently normal with no associated pain or inflammation. Cast analysis was done, which revealed no arch length-tooth size discrepancy in upper arch and mild crowding in the lower arch. Radiographic examination (Intraoral periapical radiograph, Maxillary Occlusal radiograph, panoramic radiograph) revealed deep impaction of 11 in the alveolar bone. Tooth like mass was present inferior to the impacted 11 (Figure 2). It was apparent that the mass was hindering the eruption of 11 . A provisional diagnosis of compound odontoma was made based on clinical and radiographic picture. 


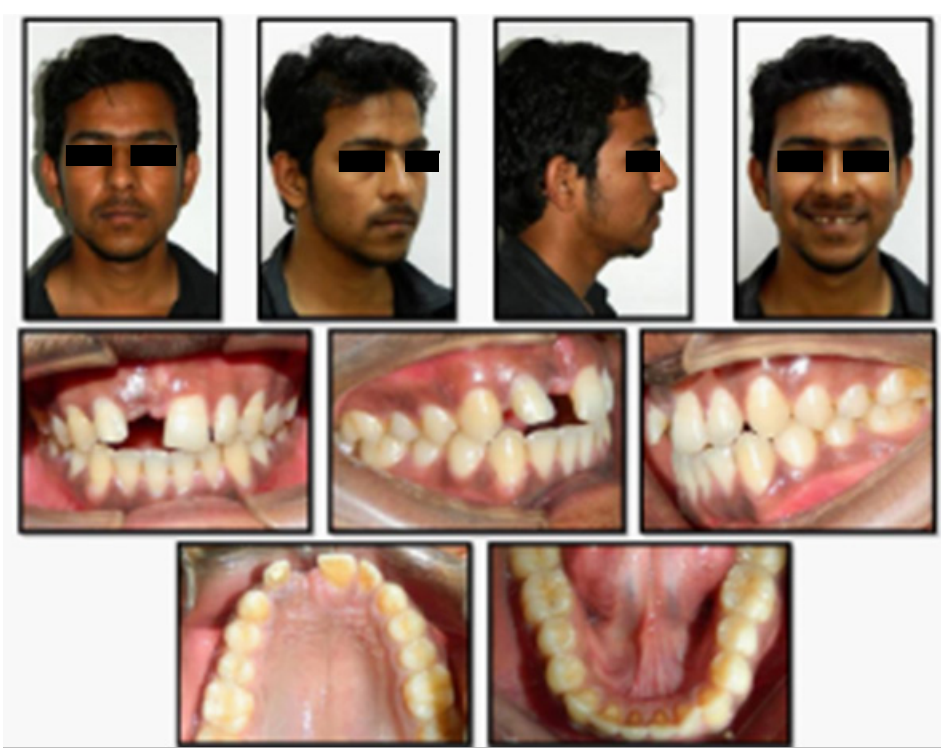

Figure I Pretreatment Photographs.
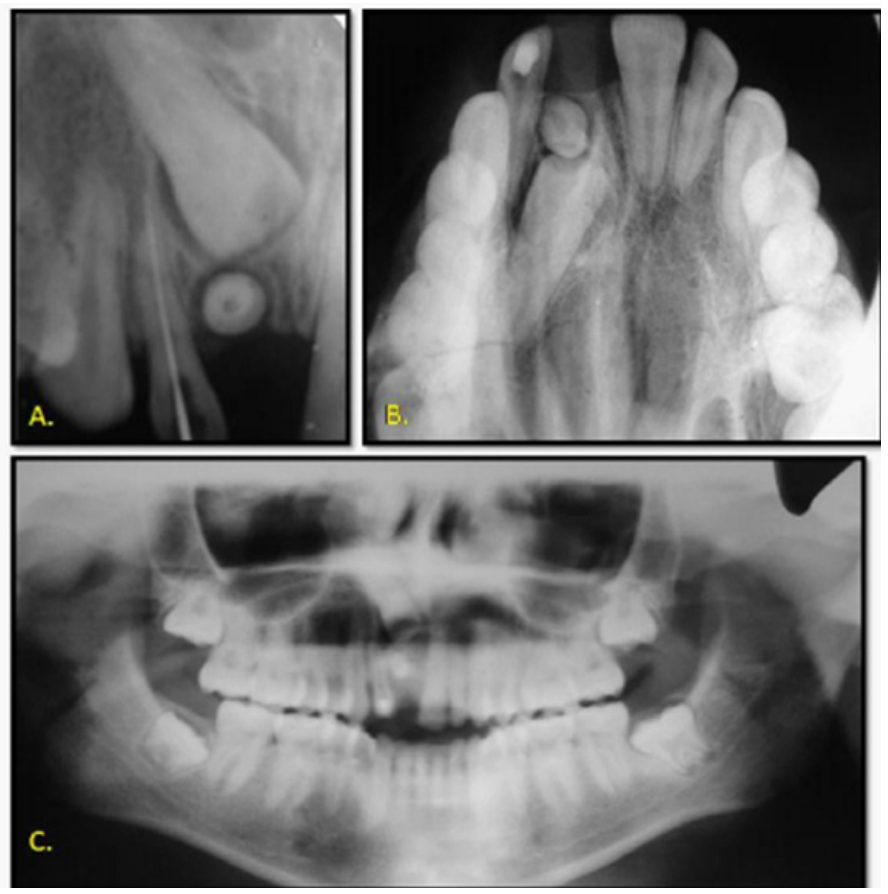

Figure 2 Pretreatment Radiogarphs.

\section{Treatment objectives:}

a. Alignment and leveling of arches.

b. Surgical removal of odontome.

c. Exposure of impacted incisor, bonding of the impacted tooth, followed by orthodontic traction.

d. Improve facial esthetics and patient's smile

e. Restorative rehabilitation of 12

\section{Treatment alternatives}

The following are three possible treatment alternatives:

a. Surgical removal of the Odontome and impacted 11 followed by prosthodontic rehabilitation with a fixed partial denture or replacement of 11 with an implant.

b. Removal of the Odontome and extraction of impacted 11 . Closure of the space, by the substitution of 21 for the central incisor with esthetic restoration of the upper right lateral incisor and canine.

c. Surgical removal of the Odontome, surgical exposure of impacted 11 followed by extrusion with low force orthodontic traction of the impacted central incisor.

\section{Treatment progress}

The treatment alternatives were considered and discussed with the patient, an informed consent was taken, and the patient chose surgical exposure and extrusion of central incisor. Pre adjusted edgewise appliance was placed, and the wires were progressed from 0.016 " NiTi to 0.021 " x 0.025 " stainless steel. Surgical exposure of the site was done with a full thickness mucoperiosteal flap. The toothlike mass was removed and sent for histopathology to confirm the diagnosis of odontoma. The crown of central incisor was exposed adequately; a lingual button was bonded on its buccal surface under proper isolation. A 0.010 " ligature wire was tied and an eyelet formed with the ligature wire was left emerging through the mucosa as the flap was sutured back (Figure 3). Orthodontic extrusion was done using light forces in the range of $80-90 \mathrm{~g}$ using NiTi coil spring. In 9months extrusion of the central incisor and alignment was achieved (Figure 4). After finishing, debonding was done followed by placement of fixed lingual retainer. The active treatment took was 16 months. The maxillary right central incisor was brought into an acceptable position with no radiographic signs of periodontal pathology or unacceptable root resorption. The overall treatment result was good, the patient's esthetics improved (Figure 5). 


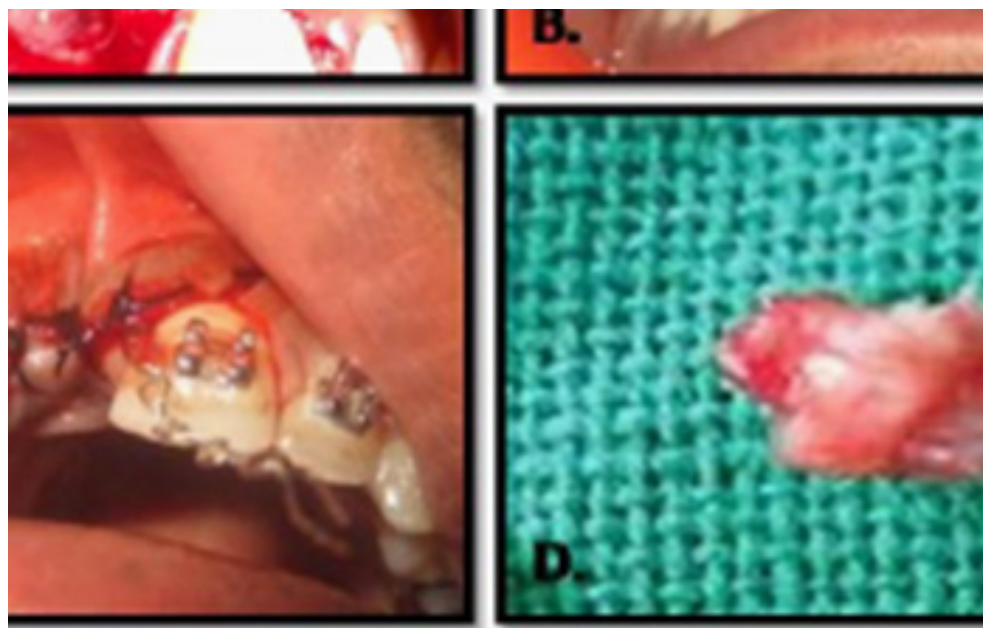

Figure 3 Surgical Procedure.

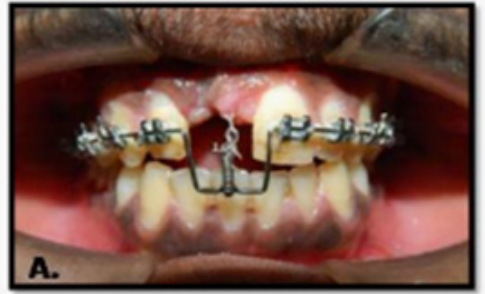

After 1 week of surgical exposure

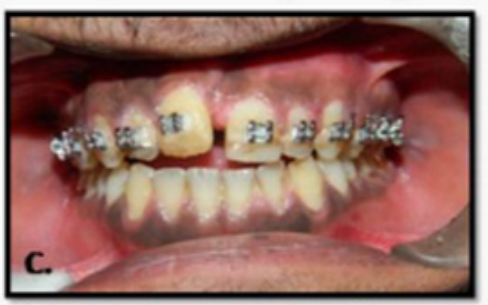

Incisor position after 7 months

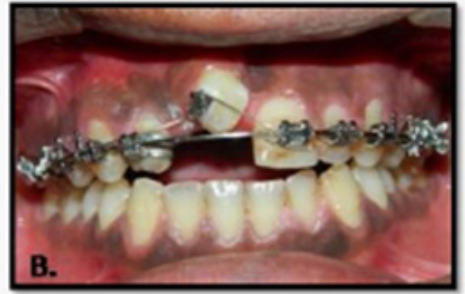

Incisor position after 5 months

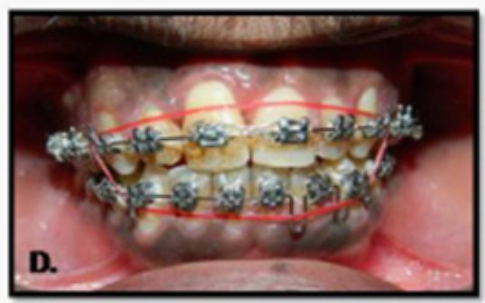

Incisor position after 9 months

Figure 4 Progress of orthodontic treatment.

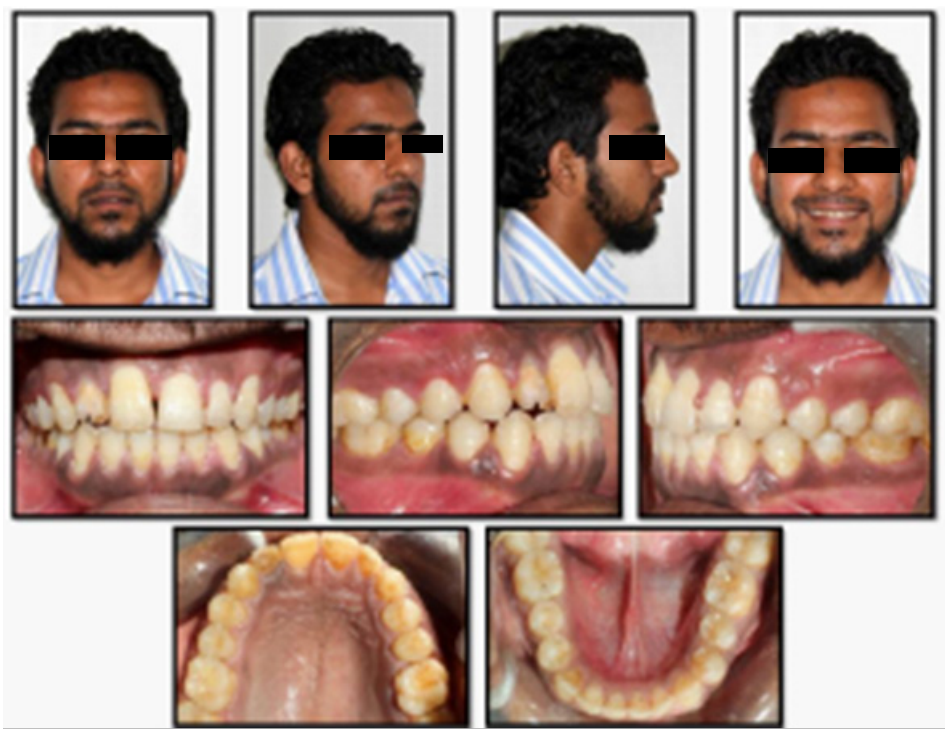

Figure 5 Post-treatment results.

Citation: Dhiman S, Khan S, Maheshwari S, et al. Orthosurgical management of odontome-associated maxillary central incisor impaction.J Dent Health Oral Disord Ther. 20I5;2(I):3-6. DOI: 10.15406/jdhodt.20I5.02.00034 


\section{Discussion}

Impacted teeth may pose problems of socialization, impaired function and speech which must be managed with careful treatment planning. Impaction may be due to the tooth size arch length deficiency, previous trauma, physical obstruction such as a supernumerary tooth, odontoma, other odontogenic tumors, cysts, or scar tissue due to early loss of primary tooth. ${ }^{13,14}$ Odontomas are often found to disrupt the eruption of teeth, it may be associated with retention of deciduous teeth or may lead to mal-positioning of the permanent tooth, delayed eruption of a permanent tooth or its impaction are its sequalae. ${ }^{15-17}$

Although it has said that odontoma has a limited potential for growth, but its surgical removal is indicated as it is derived from tooth forming epithelial cells making it susceptible to cystic change, which may lead to extensive destruction of bone. ${ }^{18}$ The treatment options range from simple observation with periodic radiographs to monitor the odontoma, clinical evaluation of eruption of dentition or surgical removal. ${ }^{19-21}$

We used closed-eruption technique to prevent any attachment loss associated with forced eruption of the central incisor. ${ }^{22,23}$ In closederuption surgical technique, the flap is sutured back to its original position after placement of an orthodontic attachment on the enamel surface of an impacted tooth. As shown in the result the periodontal status of the erupted incisor was acceptable with a healthy attached gingiva and proper gingival form. However, gingival margins of 11, 21 were at different levels, so patient was referred to the Periodontic department for the needful.

It has been reported that approximately $70 \%$ of the odontomas are associated with pathologies in the adjacent teeth such as malformation, malposition, aplasia of the tooth as a whole or resorption..$^{24}$ In our patient, the right maxillary lateral incisor neighboring the odontoma was diagnosed to be non-vital, the patient did not provide any significant history of trauma to anterior maxilla or caries of 12 , the right maxillary central incisor was impacted by the presence of the odontome in its path of eruption. Patient was referred to the department of Prosthodontic for the restorative management of 12 .

\section{Conclusion}

Odontomas present as the most common type of benign odontogenic tumours affecting the jaws. They are seldom symptomatic, so their detection relies on radiographic examinations. The treatment approach of impacted maxillary teeth with odontome requires interdisciplinary management with inputs from an Orthodontist, maxillofacial surgeon, periodontist and prosthodontics. Careful treatment planning is required to manage the case.

\section{Funding}

None.

\section{Acknowledgments}

None.

\section{Conflicts of interest}

The authors declare that there was no conflict of interest.

\section{References}

1. Odontomas SE. British Dental Journal. 1937;62:177-201.

2. Eversole LR. Clinical outline of oral pathology-diagnosis and treatment, $3^{\text {rd }}$ ed, BC Decker. 2002.
3. Sreedharan S, Krishnan IS. Compound odontoma associated with impacted maxillary incisors. J Indian Soc Pedod Prev Dent. 2012;30(3):275-278.

4. Iatrou I, Vardas E, Theologie-Lygidakis N, et al. A retrospective analysis of the characteristics, treatment and follow-up of 26 odontomas in Greek children. J Oral Sci. 2010;52(3):439-447.

5. Mcdonald J. Dentistry for the child and adolescent. $8^{\text {th }}$ ed, Elsevier, 2007;164-165pp

6. Abbott PV, Gregory PJ. Complicated crown fracture of an unerupted permanent tooth - a case report. Endod Dent Traumatol. 1998;14(1):4856.

7. Satish V, Prabhudevi MC, Sharma R. Odontome: a brief overview. International Journal of Clinical Pediatric Dentistry. 2011;4(3):177-185.

8. Hitchin AD. The aetiology of the calcified composite odontomes. British Dental Journal. 1971;130:475-482.

9. Ferrer Ramirez MJ, Silvestre Donat FJ, EstellesFerriol E, et al. Recurrent infection of a complex odontoma following eruption in the mouth. Med Oral. 2001;6(4):269-275.

10. BatraPuneet, Gupta Shweta, Rajan Kumar, et al. Odontome Diagnosis and Treatment. A Case Report. 2003;19:73-76.

11. Kramer IRH, Pindborg JJ, Shear M. Histological Typing of Odontogenic Tumors. (2nd Edn) Springer (WHO International Histological Classification of Tumors), Berlin, Germany. 1992.

12. Cohen DM, Bhattacharyya I. Ameloblastic fibroma, ameloblastic fibro-odontoma, and odontoma. Oral Maxillofac Surg Clin North Am. 2004;16(3):375-384.

13. Yeung $\mathrm{KH}$, Cheung RC, Tsang MM. Compound odontoma associated with an unerupted and dilacerated maxillary primary central incisor in a young patient. Int J Paediatr Dent. 2003;13(3):208-212.

14. Yassin OM. Delayed eruption of maxillary primary cuspid associated with compound odontoma. J Clin Pediatr Dent. 1999;23(2):147-149.

15. Hidalgo-Sanchez O, Leco-Berrocal MI, Martinez-Gonzalez JM. Metaanalysis of the epidemiology and clinical manifestations of odontomas. Med Oral Patol Oral Cir Bucal. 2008;13(11):E730-E734.

16. Kaugars GE, Miller ME, Abbey LM. Odontomas. Oral Surgery, Oral Medicine, Oral Pathology. 1989;67(2):172-176.

17. Yassin OM. Delayed eruption of maxillary primary cuspid associated with compound odontoma. J ClinPediatr Dent. 1999;23(2):147-149.

18. Singh S, Singh M, Singh I, et al. Compound composite odontome associated with an unerupted primary central incisor- a rarity. $J$ Indian Soc Pedod Prev Dent. 2005;23(3):146-150.

19. Frank C. Treatment options for impacted teeth. J Am Dent Assoc. 2000;131(5):623-632.

20. Liu J, Hsiao C, Chen H, et al. Orthodontic correction of a mandibular first molar deeply impacted by an odontoma: A case report. Quintessence Int. 1997;28(6):381-385.

21. Katz R. An analysis of compound and complex odontomas. ASDC J Dent Child. 1989;56(6):445-449.

22. Becker A, Brin I, Ben-Bassat Y, et al. Closed-eruption surgical technique for impacted maxillary incisors: a postorthodontic periodontal evaluation. Am J Orthod Dentofacial Orthop. 2002;122(1):9-14.

23. Artun J, Osterberg SK, Joondeph DR. Long-term periodontal status of labially erupted canines following orthodontic treatment. $J$ Clin Periodontol. 1986;13(9):856-861.

24. Kaneko M, Fukuda M, Sano T, et al. Microradiographic and microscopic investigation of a rare case of complex odontoma. Oral Surg Oral Med Oral Pathol Oral Radiol Endod. 1998;86(1):131-134. 